\title{
Are antimony-bismuth aprons as efficient as lead rubber aprons in providing shielding against scattered radiation?
}

\section{Authors' names and affiliations:}

Safora Johansen a, b PhD, Ingrid Helen Ryste Hauge ${ }^{\text {a, c }} \mathrm{PhD}$, Peter Hogg d MPhil, Andrew England ${ }^{\mathrm{d}} \mathrm{PhD}$, Luís Lança ${ }^{\mathrm{e}, \mathrm{f}} \mathrm{PhD}$, Catherine Gunn ${ }^{\mathrm{g}} \mathrm{MBA}$, Audun Sanderud ${ }^{\mathrm{a}} \mathrm{PhD}$

${ }^{\text {a }}$ Department of Life Sciences and Health, Oslo and Akershus University College of Applied Sciences, Oslo, Norway

${ }^{\mathrm{b}}$ Department of oncology, Division of cancer Medicine, Oslo University Hospital-Radium hospital, Oslo, Norway

${ }^{\mathrm{c}}$ Department of diagnostic physics, Division of Radiology and Nuclear Medicine, Oslo University Hospital, Oslo, Norway

${ }^{\mathrm{d}}$ School of Health Sciences, University of Salford, Manchester, United Kingdom

${ }^{\text {e }}$ ESTeSL - Escola Superior de Tecnologia da Saúde de Lisboa, Instituto Politécnico de Lisboa, Portugal

${ }^{\mathrm{f}}$ Karolinska Institutet, Stockholm, Sweden

${ }^{g}$ School of Health Sciences Dalhousie University, Halifax, Canada

\section{Corresponding author:}

Ingrid Helen Ryste Hauge

E-mail address: inheha@ous-hf.no

Full postal address: Department of diagnostic physics, (Bygg 20, Gaustad), Oslo University Hospital (OUS), P. O. Box 4959 Nydalen, NO-0424 Oslo, Norway

\section{Full postal address of each affiliation and e-mail address of each author:}

E-mail address: safora.johansen@hioa.no

Full postal address: Department of Life Sciences and Health, Oslo and Akershus University College of Applied Sciences, P. O. Box 4 St. Olavs plass, NO-0130 Oslo, Norway 
E-mail address: P.Hogg@ salford.ac.uk

Full postal address: Directorate of Radiography, College of Health \& Social Care, University of Salford, Allerton Building, Frederick Road Campus, Salford M6 6PU, United Kingdom.

Andrew England

E-mail address: $\underline{\text { A.England@ } @ \text { salford.ac.uk }}$

Full postal address: Directorate of Radiography, College of Health \& Social Care, University of Salford, Allerton Building, Frederick Road Campus, Salford M6 6PU, United Kingdom.

Luís Lança

E-mail address: luis.lanca@estesl.ipl.pt

Full postal address: Escola Superior de Tecnologia da Saúde de Lisboa, Lisbon School of

Health Technology, Av. D. João II, Lote 4.69.01, Parque das Nações, 1990-096 Lisboa,

Portugal

Catherine Gunn

E-mail address: Catherine.Gunn@nshealth.ca

Full postal address: School of Health Sciences, 657 Bethune, 1276 South Park Street, Halifax, NS, B3H 2K8 Canada

Audun Sanderud

E-mail address: audun.sanderud@hioa.no

Full postal address: Department of Life Sciences and Health, Oslo and Akershus University

College of Applied Sciences, P. O. Box 4 St. Olavs plass, NO-0130 Oslo, Norway

\section{$\underline{\text { Keywords }}$}

Absorption ability, antimony-bismuth, lead aprons, lead free aprons, Primary and scatter radiation fields 


\section{$\underline{\text { Abstract }}$}

Aim: To compare the absorption ability of two lead-free aprons with a lead apron.

Method: Measure and compare the absorption ability of 3 aprons; OpaqFu bilayer apron containing bismuth and antimony, NoLead one layer apron containing antimony, and a lead apron. The measurements were repeated with and without each of the aprons present in both primary and scattered beams. The selected tube voltages were between 60 and $113 \mathrm{kVp}$ with constant mAs, a fixed field size and fixed source to object distance.

Results: No significant difference in absorption ability of the two lead-free aprons compared to the lead apron were observed when the dose was measured in the primary beam. When measurements were performed in the scatter radiation field, the absorption ability of the OpaqFu apron was 1.3 times higher than NoLead apron and nearly equal to the absorption ability of the lead apron. An increase in the difference between the OpaqFu and NoLead aprons was observed for the tube energies higher than $100 \mathrm{kVp}$ in favor of OpaqFu apron. Conclusion: It is safe to use the lead free aprons that were tested in this study in a clinical environment for the tube energy range of $60-113 \mathrm{kVp}$. 


\section{$\underline{\text { Introduction }}$}

Lead protection garments are a key radiation protection tool against ionizing radiation ${ }^{1-4}$. The variation in protection as a function of X-ray energy is well understood for lead aprons ${ }^{1}$. The disadvantages of lead aprons are toxicity ${ }^{5}$ and weight ${ }^{1,3,6-8}$. The possibility of replacing lead with other protective devices of moderate to high atomic number elements has previously been investigated ${ }^{1,3,5,9-11}$. Weight reductions of $25 \%$ can be achieved for specific energies with lead-free aprons ${ }^{9}$, but studies ${ }^{1,3}$ have shown that lead-free garments are not as effective in attenuating radiation. However, earlier studies have concluded that most new-generation lead-free aprons provide sufficient protection and are comparable to lead aprons ${ }^{5,9}$. Bismuthantimony is one of the materials used as a shielding material for protecting the fetus in CT examinations ${ }^{12,13}$. The same lead-free material used by different manufacturers varies significantly in attenuation capabilities ${ }^{11}$. It is therefore necessary to measure and verify the $\mathrm{X}$-ray attenuation performance of protective apron materials from each manufacturer prior to clinical use ${ }^{11}$. A healthcare professional is seldom subjected to the direct radiation beam. Most often the healthcare professional stands beside the imaging table where the patient is positioned, and is subjected to scattered radiation. Therefore, the protection capability of aprons from scattered radiation must also be evaluated. To our knowledge no studies have investigated the x-ray absorption ability of antimony-bismuth aprons in a clinical environment or have directly compared their shielding effect to lead-equivalent aprons. Therefore, the aim of this study is to evaluate the $\mathrm{x}$-ray absorption abilities of one commercially available lead apron and compare it with two commercially available aprons made of different combinations of antimony and bismuth. This comparison will be made at different $\mathrm{x}$-ray energies for both primary and scattered beam radiation. 


\section{Materials and methods}

One lead, and two lead-free aprons were tested for x-ray absorption ability in both the primary and scattered radiation fields. The method for the measurements performed in this study (with and without the aprons) is based on methods described in previous studies ${ }^{5,14-16}$.

\section{Apron description}

The lead-free aprons were from Scanflex Medical AB (Täby, Sweden): “Opaque Fusion ${ }^{\circledR}$ $0.35 \mathrm{~mm}$ " (“OpaqFu") and "No Lead $0.35 \mathrm{~mm}$ " ("NoLead"). They consisted of a combination of the two metals - antimony and bismuth. Although OpaqFu and NoLead are made from the same two metals, OpaqFu has been manufactured using a so-called bilayer technique with thinner layers that reduce the weight of the apron ${ }^{9}$. The lead apron was manufactured by Mavig GmbH (Munich, Germany): "Pb 0.35 mm” ("Pb”). All aprons were checked for defects prior to use. In order to encompass the use of aprons at higher energies (over 100 $\mathrm{kVp}$ ), aprons with a specified lead equivalent thickness of $0.35 \mathrm{~mm}$ were selected, as recommended by IPEM ${ }^{17}$.

\section{Measurements in the primary radiation field}

A Siemens Multix TOP x-ray unit (Siemens Healthcare GmbH, Erlangen, Germany) with a tungsten anode and inherent filtration of $2.5 \mathrm{~mm} \mathrm{Al}$ at $70 \mathrm{kVp}$ was used to generate 17 different tube voltages between $60 \mathrm{kVp}$ and $113 \mathrm{kVp}$, in steps of 2-4 kVp. For measurements in the primary and scatter beam a tube current-time product of $10 \mathrm{mAs}$ was used both with and without an apron present in the radiation field (Figure 1). Dose measurements in the primary beam were performed using a Mult-O-Meter (MOM) (Unfors, Instruments AB, Billdal, Sweden). To minimize random error, dose measurements were repeated 3 times and then averaged; no difference in these dose measurements were observed.

Prior to data collection, variability of the dose measurement for the selected tube voltages and a variation coefficient for primary radiation field $(2.8 \%)$ and scatter radiation field $(3.0 \%)$ was determined ${ }^{15,18}$.

The difference in effective energy between the primary beam and scattered radiation was also measured. The difference ranged from $-9.5 \%$ to $-13.3 \%$, for selected tube voltages $60 \mathrm{kVp}$ and $113 \mathrm{kVp}$, respectively. Furthermore, the lead equivalent thickness of the lead-free aprons in the direct radiation beam was also determined, in order verify whether they fulfilled the lead-equivalent value given by the manufacturer ${ }^{3,16}$. The results showed that the lead-free aprons fulfilled the equivalent lead thickness of $0.35 \mathrm{~mm}$ specified by the manufacturer. 
A short source to image distance (SID) of $60 \mathrm{~cm}$ was used to achieve a high intensity of radiation behind each of the aprons. The choice of SID does not resemble a clinical setting; however, the selection of SID should not influence the energy of the radiation field per se. Field size of $30 \times 30 \mathrm{~cm}$ and a $60 \mathrm{~cm}$ source to object distance were used.

a)

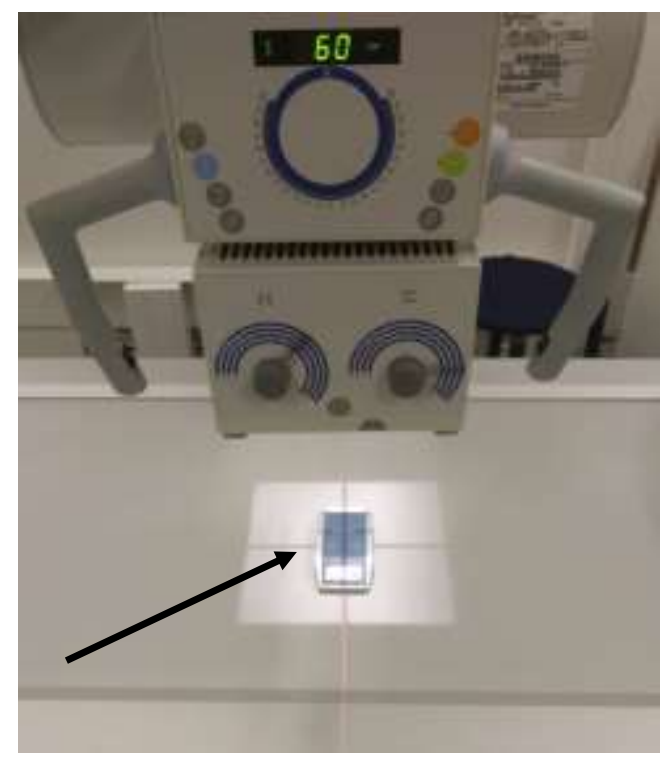

b)

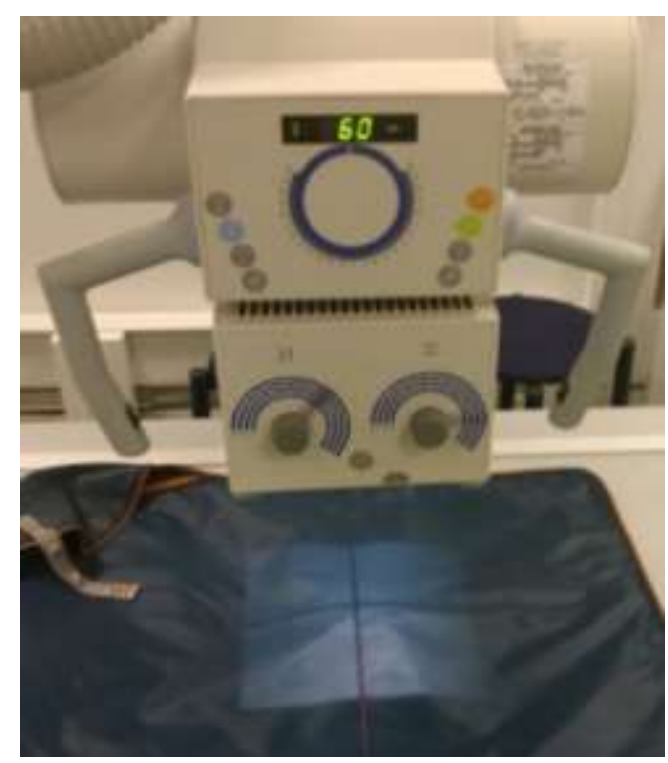

\section{Figure 1.}

a) An image of the MOM detector as seen without the protective garment.

b) The MOM detector placed in the radiation field (same location as in Fig 1a) behind the protective garment.

\section{Measurements in the scattered radiation field}

Scattered radiation was generated by irradiating an anthropomorphic pelvis phantom

(Radiology Support Devices Inc., Carson, CA, USA) on the imaging table within the direct beam. A second anthropomorphic (thorax) phantom (Radiology Support Devices Inc., USA) was placed close to the pelvis phantom on the table (Figure 2a), to mimic the healthcare professional standing next to a patient. A Direct Dosimeter was used (EDD-30) (Unfors, Instruments $\mathrm{AB}$, Billdal, Sweden) to measure the scattered radiation from the pelvic phantom within the primary radiation field (Figure 2a) and the absorbed dose behind each apron (Figure 2b). To measure the radiation dose, the EDD-30 probe was kept in the same position as in Figure $2 \mathrm{a}$ and then covered by an apron (Figure $2 b$ ).

A dose measurement behind each of the three aprons and also without any apron was conducted for each of the tube voltages. For each exposure, a large collimation field size (30 x $30 \mathrm{~cm}$ ) was applied. A SID equal $60 \mathrm{~cm}$ and a source to object distance (SOD) of $25 \mathrm{~cm}$ was 
used (Figure 2). These parameters contributed to maximize the amount of scattered radiation in order to enhance the accuracy of the dose measurements behind the aprons.

a)

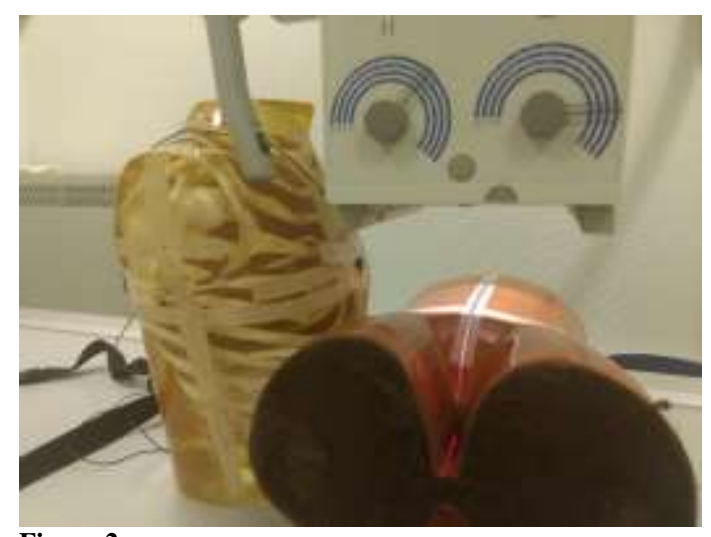

b)

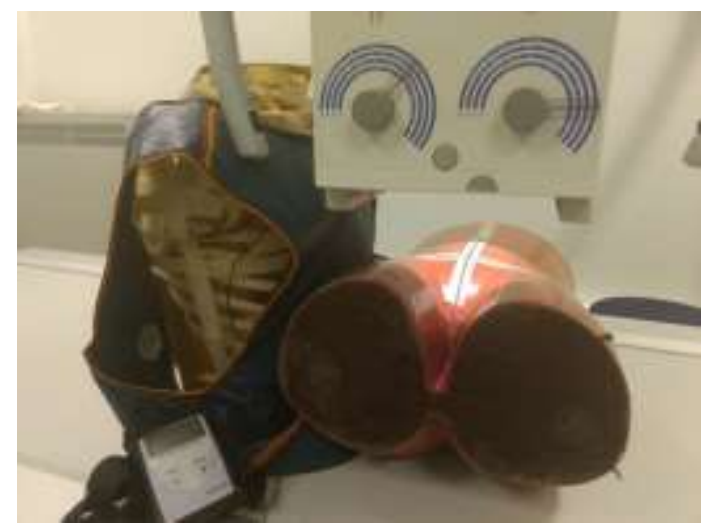

a) An image of EDD-30 dose probe (marked with an arrow) as seen without the protective garment.

b) The EDD-30 dose probe was placed in the radiation field (same location as in image a)) behind the protective garment.

The EDD-30's sensitivity ranges from 10 nGy to 9999 Gy, and dose rates between $10 \mathrm{nGy} / \mathrm{s}$ and $0.6 \mathrm{mGy} / \mathrm{s}$.

\section{Statistical testing}

To assess differences between the absorption ability of the lead apron vs the lead-free aprons, a homoscedastic two-tailed independent student t-test (Two-sample qual vaiance) was employed. A value of $<0.05$ was considered to be statistically significant. Mean, median, minimum, maximum dose and differences between the absorbed dose, the ratio of dose absorption in the 3 employed aprons, and standard deviation were also calculated.

\section{$\underline{\text { Results }}$}

\section{Measurements in the primary radiation field}

Table 1 and Figure 1 provide the registered dose by MOM in the primary radiation field with and without the 3 aprons for the range of $\mathrm{kVp}$ values. For $\mathrm{kVps}<90$ and between 100-113 the lead apron shows a slightly higher absorption ability than the other aprons. For the majority of the $\mathrm{kVp}$ values between $\geq 90$ and $<102$ the aprons containing antimony and bismuth and particularly the OpaqFu showed a higher dose absorption compared to the lead apron. However, the differences in the cohort-based median of $-2.4,-0.8$ and mean of $-3.7,-1.9$ ( $\mu \mathrm{Gy}$ ) show higher radiation absorption by the lead apron when compared to NoLead and 
OpqaFu aprons, respectively. These differences are not significant for the range og $\mathrm{kVp}$ values used in this study (Table 1) or for kVps values of 60 to 90, 90 to 100 and 100-113 (pvalues not shown). The differences are significant only when the absorption ability is compared with the measurements performed in the absence of an apron compared with 3 different aprons as shown in Table 1. Furthermore, our results report that the absorption ability of OpaqFu is one time higher than the absorption ability of the NoLead apron (Table 1). This difference is not statistically significant $(\mathrm{p}=0.540)$.

\section{Measurements in the scatter radiation field}

The results of the dose measurement in the scatter radiation field show that the OpaqFu has an identical or slightly higher absorption ability than the lead and NoLead aprons (Table 2 and Figure 2). However, this difference is not statistically significant as presented in the Table 2. The median and mean absorption ability of OpaqFu is 0.7 and $0.8 \mu \mathrm{Gy}$ higher when compared to the NoLead apron. However, this difference is not statistically significant. The absorption ability ratio of OpaqFu is 1.3 and 1.0 times higher than the NoLead and lead aprons, respectively. The radiation absorption ability of the NoLead apron worsens when the tube voltage increases as illustrated in figure 2.

\section{$\underline{\text { Discussion }}$}

The absorption ability of the lead apron was compared with 2 lead-free aprons in both primary and scatter radiation fields. Overall, no significant differences in absorption ability was observed between the selected aprons in both primary and scatter radiation fields. However, for some selected tube energies the OpaqFu apron performed better than the NoLead and lead aprons when the dose is measured in the scatter radiation field. This difference is more apparent when comparing the OpaqFu with the NoLead apron.

\section{The aprons' protective effect in the primary radiation field}

In the energy range of 93-96 and 93-100, the NoLead and OpaqFu aprons have slightly higher absorption abilities than the lead apron, respectively (Table 2). This indicates that both aprons have one or more materials with a K-edge that peaks in attenuation for a primary beam near $90 \mathrm{kVp}$. The bismuth in the OpacFu (with a K-edge of $90.5 \mathrm{keV}$ ), makes it just as effective as lead (with a K-edge of $88 \mathrm{keV}$ ) in attenuating the radiation from the X-ray tube. The antimony in the NoLead apron on the other hand has a K-edge of $30.5 \mathrm{keV}$. This K-edge is too low for the energies applied in this study, thus making it less effective in attenuating radiation through 
the photoelectric effect for tube voltages in the range $60-113 \mathrm{kVp}$. Another difference between the OpaqFu and NoLead is that OpaqFu has a bilayer structure, making it apparently more effective in radiation absorption. Multilayer attenuators "harden" the X-ray beam by narrowing the X-ray energy spectra ${ }^{9}$.

\section{The aprons' protective effect against scattered radiation}

For scattered radiation the OpaqFu apron seems to be just as effective as the lead apron in shielding against scattered radiation at any energy. This can be due to the fact that more of the scattered radiation from a $120 \mathrm{kVp}$ beam lies near the K-edge of the materials used in the construction of lead-free aprons ${ }^{1}$. So even if the absorption ability for the primary radiation of the OpaqFu apron was shown to be less than the absorption ability of the lead apron for tube voltages higher than $100 \mathrm{kVp}$, the absorption ability for the scattered radiation indicates that due to a shift in the scattered radiation towards lower energies, the OpaqFu apron can be just as effective as lead in shielding against radiation up to a tube voltage of $113 \mathrm{kVp}$.

\section{$\underline{\text { Conclusion }}$}

The results in this study demonstrate that for scattered radiation a bilayer apron (OpaqFu) has slightly better or equal shielding effects for tube voltages 60 to $113 \mathrm{kVp}$ when compared to the absorption ability of the NoLead and lead aprons. For kVp values between $60-113 \mathrm{kVp}$, healthcare professionals should consider using the OpaqFu apron instead of the lead apron due to its improved x-ray protection capability.

\section{$\underline{\text { References }}$}

1. Jones AK, Wagner LK. On the (f)utility of measuring the lead equivalence of protective garments. Med Phys. Jun 2013;40(6):063902.

2. Schueler BA. Operator shielding: how and why. Techniques in vascular and interventional radiology. Sep 2010;13(3):167-171.

3. Finnerty M, Brennan PC. Protective aprons in imaging departments: manufacturer stated lead equivalence values require validation. Eur Radiol. Jul 2005;15(7):1477-1484.

4. Le Heron J, Padovani R, Smith I, Czarwinski R. Radiation protection of medical staff. Eur J Radiol. Oct 2010;76(1):20-23.

5. Cetin H, Yurt A, Yuksel SH. The Absorption Properties of Lead-Free Garments for Use in Radiation Protection. Radiat Prot Dosimetry. Feb 152016.

6. Moore B, vanSonnenberg E, Casola G, Novelline RA. The relationship between back pain and lead apron use in radiologists. AJR Am J Roentgenol. Jan 1992;158(1):191-193.

7. Goldstein JA, Balter S, Cowley M, Hodgson J, Klein LW, Interventional Committee of the Society of Cardiovascular I. Occupational hazards of interventional cardiologists: prevalence of orthopedic health problems in contemporary practice. Catheter Cardiovasc Interv. Dec 2004;63(4):407-411.

8. Ross AM, Segal J, Borenstein D, Jenkins E, Cho S. Prevalence of spinal disc disease among interventional cardiologists. The American journal of cardiology. Jan 1 1997;79(1):68-70. 
9. McCaffrey JP, Mainegra-Hing E, Shen H. Optimizing non-Pb radiation shielding materials using bilayers. Med Phys. Dec 2009;36(12):5586-5594.

10. McCaffrey JP, Shen H, Downton B, Mainegra-Hing E. Radiation attenuation by lead and nonlead materials used in radiation shielding garments. Med Phys. Feb 2007;34(2):530-537.

11. McCaffrey JP, Tessier F, Shen H. Radiation shielding materials and radiation scatter effects for interventional radiology (IR) physicians. Med Phys. Jul 2012;39(7):4537-4546.

12. Chatterson LC, Leswick DA, Fladeland DA, Hunt MM, Webster ST. Lead versus bismuth-antimony shield for fetal dose reduction at different gestational ages at CT pulmonary angiography. Radiology. Aug 2011;260(2):560-567.

13. Chatterson LC, Leswick DA, Fladeland DA, Hunt MM, Webster S, Lim H. Fetal shielding combined with state of the art CT dose reduction strategies during maternal chest CT. Eur J Radiol. Jul 2014;83(7):1199-1204.

14. Hubbert TE, Vucich JJ, Armstrong MR. Lightweight aprons for protection against scattered radiation during fluoroscopy. AJR Am J Roentgenol. Nov 1993;161(5):1079-1081.

15. Mori H, Koshida K, Ishigamori O, Matsubara K. Evaluation of the effectiveness of X-ray protective aprons in experimental and practical fields. Radiological physics and technology. Jan 2014;7(1):158166.

16. Schopf T, Pichler T. Radiation Protection Clothing in X-Ray Diagnostics - Influence of the Different Methods of Measurement on the Lead Equivalent and the Required Mass. Rofo. Aug 2016;188(8):768775 .

17. Institute of physics and engineering in medicine (IPEM). Medical and Dental Guidance Notes. Paragraph 3.119 01/01/2002 2002.

18. National Institute of Standards and Technology. X-ray mass attenuation coefficients lead. http://physics.nist.gov/PhysRefData/XrayMassCoef/ElemTab/z82.html.

\section{Figures and Tables legend:}

Figure 1: The absorption ability of 2 non-lead aprons and a lead apron in the primary field

Figure 2: The absorption ability of 2 non-lead aprons and a lead apron in the scatter field

Table 1: Dose (D) measurements ( $\mu \mathrm{Gy}$ ) for the primary radiation field with no apron (NP),

Lead (LE), NoLead (NL), OpaqFu (OP) when mAs=10

Table 2: Dose (D) measurements ( $\mu \mathrm{Gy}$ ) for the scatter radiation field with no apron (NP), Lead (LE), NoLead (NL), OpaqFu (OP) when mAs=10 